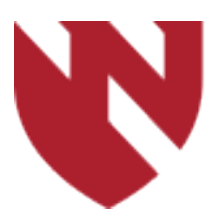

December 2019

\title{
Do Pre-operative Emotional and Mental Rand SF-36 Scores Predict Outcomes after Total Hip and Knee Arthroplasty?
}

\author{
Erik Bowman \\ University of Nebraska Medical Center \\ Curtis Hartman \\ University of Nebraska Medical Center \\ Kevin Garvin \\ University of Nebraska Medical Center \\ Beau Konigsberg \\ University of Nebraska Medical Center \\ Liz Lyden \\ University of Nebraska Medical Center
}

See next page for additional authors

Tell us how you used this information in this short survey.

Follow this and additional works at: https://digitalcommons.unmc.edu/gmerj

Part of the Higher Education Commons, and the Medicine and Health Sciences Commons

\section{Recommended Citation}

Bowman, E., Hartman, C., Garvin, K., Konigsberg, B., Lyden, L., , Wardyn, S. Do Pre-operative Emotional and Mental Rand SF-36 Scores Predict Outcomes after Total Hip and Knee Arthroplasty?. Graduate Medical Education Research Journal. 2019 Dec 13; 1(1).

https://digitalcommons.unmc.edu/gmerj/vol1/iss1/78

This Conference Proceeding is brought to you for free and open access by DigitalCommons@UNMC. It has been accepted for inclusion in Graduate Medical Education Research Journal by an authorized editor of DigitalCommons@UNMC.For more information, please contact digitalcommons@unmc.edu. 
Do Pre-operative Emotional and Mental Rand SF-36 Scores Predict Outcomes after Total Hip and Knee Arthroplasty?

\section{Creative Commons License}

\section{(c) (1) $\Theta(9$}

This work is licensed under a Creative Commons Attribution-Noncommercial-No Derivative Works 4.0 License.

Authors

Erik Bowman, Curtis Hartman, Kevin Garvin, Beau Konigsberg, Liz Lyden, and Seth Wardyn 


\section{Three Cases of Laryngeal Histoplasmosis in The Midwestern United States}

John Blecha, Christopher Bingcang, Justin Oltman

Mentor: Christopher Bingcang

Program: Otolaryngolgy

Introduction: Laryngeal histoplasmosis is a rare entity, with $<100$ case reports in the literature. Patients with laryngeal involvement often report hoarseness, mucosal ulcerations, dysphagia, and odynophagia. Laryngeal lesions caused by histoplasmosis are highly variable in both location and appearance, and can be easily misdiagnosed as either malignancy, tuberculosis, or granulomatous diseases.

Case description: We present a series of three cases of laryngeal histoplasmosis. All patients presented with new onset voice changes, odynophagia, and dysphagia. All patients were immunosuppressed secondary to treatment of medical co-morbidities with two treated for Crohn's disease and one receiving chemotherapy for prostate cancer. Upon endoscopic evaluation, ulcerative lesions and thickening were found in all three patients. Those with Crohn's disease had diffuse supraglottic disease, while the patient receiving chemotherapy had a unilateral true vocal fold lesion. These lesions were suspicious for malignancy and biopsies were obtained. Biopsies returned negative for malignancy. In one patient with Crohn's, a biopsy was indeterminate, and subsequently developed a bowel perforation and was diagnosed with disseminated histoplasmosis on surgical pathology. One patient was diagnosed after fungal cultures grew Histoplasma at four weeks. Increased suspicion led to diagnosis via Gomori Methenamine Silver Stain in another. All patients were subsequently followed by infectious disease, started on antifungals, and symptoms resolved.

Discussion: These cases illustrate the high index of suspicion required to make the diagnosis of laryngeal histoplasmosis, especially in the Midwestern United States. Diagnosis may be made quickly and costeffectively with proper staining. Delayed diagnosis may lead to dissemination, increased morbidity, and mortality.

https://doi.org/10.32873/unmc.dc.gmerj.1.1.077

\section{Do Pre-Operative Emotional and Mental Rand SF-36 Scores Predict Outcomes after Total Hip and Knee Arthroplasty? \\ Erik Bowman, Curtis Hartman, Kevin Garvin, Beau Konigsberg, Liz Lyden, Seth Wardyn}

Mentor: Curtis Hartman

Program: Orthopaedic Surgery

Introduction: Preoperative psychosocial risk factors have been shown to correlate with poor outcomes in the short term after total knee arthroplasty, however, outcome measures and function have not been correlated to objective emotional and mental health data. The purpose of this single center retrospective study was to determine if the emotional and mental health scores on the short form 36 (SF36) correlated with outcomes.

Objective: To determine if pre-operative emotional and mental health scores on the
Rand SF-36 predict outcomes after total knee and hip arthroplasty.

Methods: Retrospective review of 733 total hip and knee arthroplasties performed at UNMC over a 2 year period. Role emotional pre-surgery and mental health pre-surgery were used to predict the following outcomes: all WOMAC score post-surgery, the change in WOMAC scores (post-pre), disposition (skilled nursing facility verses home), intervention within 90 days and hospital/ER visit (based on 1st visit).

Results: The results of this data showed a statistically significant correlation in preoperative emotional and mental health scores with hospital length of stay postoperative pain scores, physical function, total WOMAC score and disposition to skilled nursing facility or home. A multivariate analysis controlling for age, gender and BMI, however, showed that only general mental health scores are weakly associated with hospital length of stay postoperative pain scores, hysical function, and total WOMAC score.

Conclusion: This study shows that there is a statistically significant weak correlation in objective SF36 emotional and mental health scores and patient outcomes.

https://doi.org/10.32873/unmc.dc.gmerj.1.1.078

\section{BMI as a Risk Factor for Early Dislocation of Total Shoulder Arthroplasty}

David J. Kusin, Joshua A. Ungar, Kaeli K. Samson, Matthew J. Teusink

Mentor: Matthew Teusink

Program: Orthopaedic Surgery

Background: Dislocation of total shoulder arthroplasty has an incidence as high as $31 \%$. Obesity is one of many proposed risk factors, but no consensus exists on this relationship. The purpose of this study was to determine whether the relationship between BMI and dislocation of total shoulder arthroplasty.

Methods: The NSQIP database was used to identify patients older than 50 who underwent anatomic or reverse total shoulder arthroplasty between the years 2012-2016 for primary or secondary osteoarthritis, post-traumatic arthritis, or cuff tear arthropathy. Patients requiring reoperation or readmission for dislocation were identified by CPT code. The relationship between WHO BMI classification and dislocation was assessed.

Results: 9,382 patients were identified. $46 \%$ were male and $54 \%$ female, with an average age of 68.7 (range 50-90) and average BMI of 31.2 (range 15.1-79.3). There were only 24 dislocation events within the first 30 days after the procedure $(0.26 \%)$. Ten of $24(42 \%)$ occurred after discharge. $70 \%$ of cases $(17$ of 24) required an open procedure. Underweight patients $(\mathrm{BMI}<18.5)$ experienced the highest dislocation rate $(1 / 50,2.00 \%)$, whereas overweight patients (BMI 25-29.9) experienced the lowest dislocation rate $(3 / 3069,0.1 \%)$.

Conclusion: The rate of dislocation of total shoulder arthroplasty in the acute postoperative period differs across categories of BMI. However, there does not appear to be a linear association between BMI and risk of dislocation of total shoulder arthroplasty. Further studies are required to elucidate the risk factors for total shoulder dislocation. https://doi.org/10.32873/unmc.dc.gmerj.1.1.079 